La participación gubernamental en el diseño de estrategias comerciales para la industria del calzado en México, desde la perspectiva de los derechos económicos, sociales y culturales

Governmental participation in the design of foreign trading strategies for the footwear industry in Mexico, from a perspective of economic, social and cultural rights

Autor: Gabriela Aguado Romero

DOI: https://doi.org/10.25058/1794600X.1915

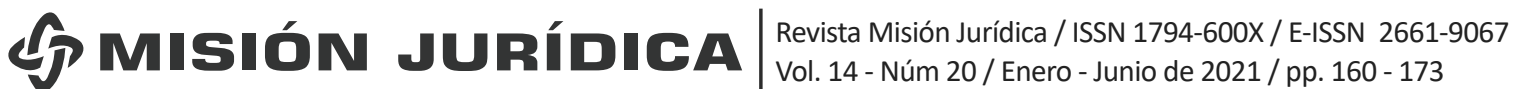




\title{
LA PARTICIPACIÓN GUBERNAMENTAL EN EL DISEÑO DE ESTRATEGIAS COMERCIALES PARA LA INDUSTRIA DEL CALZADO EN MÉXICO, DESDE LA PERSPECTIVA DE LOS DERECHOS ECONÓMICOS, SOCIALES Y CULTURALES*
}

\author{
Governmental participation in the design of foreign trading \\ strategies for the footwear industry in Mexico, from a \\ perspective of economic, social and cultural rights
}

\section{Participação do governo no desenho de estratégias comerciais para a indústria calçadista no México, desde a perspectiva dos direitos econômicos, sociais e culturais}

Gabriela Aguado Romero ${ }^{a}$ aguadogabriela@hotmail.com

Fecha de recepción: 15 de junio de 2020 Fecha de revisión: 30 de junio de 2020

Fecha de aceptación: 20 de julio de 2020

Para citar este artículo:

Aguado Romero, G. (2021). La participación gubernamental en el diseño de estrategias comerciales para la industria del calzado en México, desde la perspectiva de los derechos económicos, sociales y culturales. Revista Misión Jurídica, 14, (20), 160 -173.

\section{RESUMEN}

La industria del calzado se ha desarrollado en la zona de El Bajío mexicano, siendo Guanajuato el Estado pionero; sin embargo, los Estados de Querétaro y Aguascalientes pretenden emularlo. En el pasado, el proteccionismo estatal otorgó estabilidad al sector no obstante, el cambio de modelo económico a nivel mundial obligó a la nación a adoptar una nueva corriente económica, con la apertura de fronteras comerciales en beneficio de los grandes productores de calzado, pero no de los medianos y pequeños, algunos de ellos han sabido sortear los vaivenes que dictan los mercados internacionales, pero otros desaparecieron. Hoy se presenta un nuevo paradigma, el

\footnotetext{
* El texto es resultado parcial del proyecto de investigación "Esquema de empresas certificadas, bajo la modalidad de operador económico autorizado: herramienta o barrera para las pequeñas y medianas empresas de la industria del calzado de la zona de El Bajío mexicano, desde la perspectiva de los derechos económicos, sociales y culturales". La responsable del proyecto es la Dra. Gabriela Aguado Romero, profesora investigadora de la Universidad Autónoma de Querétaro, México. La investigación cuenta con financiamiento del Fondo para el Fortalecimiento de la Investigación FOFI 2018 - 2020, registro aprobado FDE201805.

a. Licenciada en Derecho con Especialidad en Derecho Fiscal y en Derecho Notarial, Maestría y Doctorado en Derecho por la Universidad Autónoma de Querétaro, México. Profesora e investigadora de tiempo completo de la Facultad de Derecho (UAQ), con Reconocimiento Perfil Deseable PRODEP, integrante del Cuerpo Académico Consolidado "Derechos humanos y globalización" CACUAQ-128 Facultad de Derecho (UAQ), miembro del Sistema Nacional de Investigadores del Consejo Nacional de Ciencia y Tecnología, SNI Nivel I. ORCID: https://orcid.org/0000-0003-3733-6459.
} 
escenario mundial ha cambiado, en medio de pandemias, el anuncio de la Cuarta Revolución Tecnológica y una guerra comercial entre China y Estados Unidos, el Estado mexicano debe replantearse el rol que debe desempeñar, pues tal parece que su participación no ha de ser la de un Estado intervencionista, pero sí se requiere de su cooperación directa en cumplimiento a lo que disponen los derechos económicos, sociales y culturales, protegidos por diversos tratados internacionales y regionales, así como en la Constitución mexicana, cumpliendo con los compromisos adquiridos en los acuerdos sobre esta materia, el Estado debe diseñar estrategias que generen competencia seria y frontal, con el objetivo de lograr que pequeños y medianos manufactureros consoliden la exportación de su calzado.

\section{PALABRAS CLAVE}

Industria del calzado; estrategias comerciales; gobierno; derechos económicos; sociales $\mathrm{y}$ culturales.

\section{ABSTRACT}

The footwear industry has developed in the area of the Mexican Bajío, Guanajuato, which is the pioneering State, however, the States of Querétaro and Aguascalientes are trying to emulate its example. In the past, State protectionism gave stability to the sector. However, the change in the economic model worldwide forced our nation to adopt a new economic trend, that led to the opening of commercial borders and benefited large footwear producers, but not medium and small-sized ones, some of them have managed to get around the ups and downs dictated by international markets, but others disappeared. Nowadays a new paradigm is emerging, the world scenario is different, in the midst of a pandemic, the announcement of the Fourth Technological Revolution and a trade war between China and the United States, the Mexican State must rethink the role it should play, as it seems its participation should not be that of an interventionist State, but its direct cooperation is required in compliance with the provisions of economic, social and cultural rights, protected by various international and regional treaties, as well as in the Mexican Constitution, in compliance with the commitments made in the agreements on this matter, the State must design strategies that generate serious and frontal competition, with the objective that small and medium sized manufacturers manage to consolidate their exports.

\section{KEYWORDS}

Footwear industry; business strategies; government; economic; social and cultural rights.

\section{RESUMO}

A indústria de calçados se desenvolveu na região mexicana de Bajío, sendo Guanajuato o Estado pioneiro; no entanto, os Estados de Querétaro e Aguascalientes procuram imitálo. No passado, o protecionismo estatal deu estabilidade ao setor, porém, a mudança no modelo econômico mundial obrigou o país a adotar uma nova tendência econômica, com a abertura das fronteiras comerciais em benefício dos grandes calçadistas, mas não dos médios e pequenos, alguns deles conseguiram superar os altos e baixos que ditam os mercados internacionais, mas outros desapareceram. Hoje um novo paradigma se apresenta, o cenário mundial mudou, em meio a pandemias, o anúncio da Quarta Revolução Tecnológica e uma guerra comercial entre a China e os Estados Unidos, o Estado mexicano deve repensar o papel que deve desempenhar, pois parece que A participação não deve ser a de um Estado intervencionista, mas sua cooperação direta é necessária em conformidade com as disposições dos direitos econômicos, sociais e culturais, protegidos por diversos tratados internacionais e regionais, bem como na Constituição mexicana, cumprindo os compromissos previstos nos acordos sobre a matéria, o Estado deve traçar estratégias que gerem concorrência séria e frontal, com o objetivo de assegurar que os pequenos e médios fabricantes consolidem a exportação de seus calçados.

\section{PALAVRAS-CHAVE}

Indústria calçadista; estratégias comerciais; governo; direitos econômicos; social e cultural.

\section{INTRODUCCIÓN}

La presente investigación tiene como objetivo demostrar que es necesario el apoyo gubernamental a través de políticas públicas que beneficien a las distintas ramas de la industria, 
siendo prioridad para esta investigación las relacionadas concretamente, con los fabricantes de calzado localizados en la región de El Bajío mexicano.

En la primera parte del artículo se analizan las demandas sociales de la población, las cuales se encuentran plasmadas en la Constitución Política, siendo una de ellas el derecho al trabajo. El desmedido crecimiento de la burocracia permitió al Estado generar una gran cantidad de puestos de trabajo, sin embargo, el proteccionismo estatal no permitió que la iniciativa privada fuera otra fuente creadora de puestos laborales.

El cambio de paradigma económico prometía la generación de empleos bien remunerados, pues se aseguraba que la inversión extranjera traería un sinfín de beneficios. Se hablaba de una gran derrama económica que, si bien su origen estaría en la cúspide, con el paso del tiempo permearía a todas las capas de la sociedad.

Luego se habla de las intenciones de la iniciativa privada, en cuanto a querer encargarse de la creación de un mejor escenario económico para la sociedad en general, a través de la generación de empleos, bajo la advertencia de que habría una mayor oferta de empleos en la medida que los empresarios tuvieran una mayor independencia para actuar en el mercado. Sin embargo, esto resultó una falacia, pues no solo no disminuyó el desempleo, sino que aumentó, al igual que el empleo informal y el subempleo, lo que se traduce en ocupaciones laborales de ínfima calidad que no le dan ningún tipo de seguridad social a quienes están inmersos en estas actividades, además no contribuyen fiscalmente, lo que impide que el gobierno lleve a cabo estrategias que permitan una mejora en el interés general.

Ahora nos enfrentamos a un nuevo paradigma, el cual se dice cambiará la manera de llevar a cabo las interrelaciones humanas, se prevé que estos cambios sean inmediatos, pues aparte de esa revolución digital que ya se ha anunciado, hay otros dos factores principales, que aceleraran aún más la revolución venidera: la guerra comercial Estados Unidos-China y, los estragos provocados por la pandemia del Covid-19.

El papel que juegue el Estado será trascendental, pues por una parte ha de tener la capacidad para ser un actor importante en el ámbito internacional y saber comportarse como un verdadero socio comercial para así influir y proporcionar a la industria nacional los mecanismos adecuados para salir a exportar. Por otro lado, debe el gobierno tomar las decisiones internas correctas, velando siempre por el cumplimiento de los derechos relativos a las condiciones económicas y sociales básicas para una vida en dignidad y libertad como así lo disponen los Derechos Económicos Sociales y Culturales (DESC) y a los que se encuentra obligado el Estado mexicano, y que a final de cuentas, guardan relación con el referido apoyo que le debe proporcionar a la industria en general, donde está incluida la industria del calzado.

\section{METODOLOGÍA}

Para estudiar el tema se utilizó la metodología de investigación cualitativa, con un paradigma epistemológico crítico. Para esto, se emplearon como técnicas de recolección de información, la revisión documental contenida en diversas fuentes primarias, que se encuentran en informes gubernamentales, que permitió identificar temas específicos relevantes para el campo de la investigación y, como fuentes secundarias se exploró la doctrina especializada relacionada con el objeto de estudio, documentos obtenidos en bases de datos académicas, bibliografía y hemerografía.

Para el presente estudio se recurrió a los métodos dogmático, deductivo, analítico y sintético, los cuales inducen a la utilización de la técnica de investigación documental. Fue necesario recurrir al método exegético en cuanto al uso de la legislación.

Las fases metodológicas fueron: una primera fase, de recolección de información y su clasificación; una segunda fase, fue el análisis de la anterior, a través de la proyección metodológica que se asumió; y una fase final, de interpretación del análisis y construcción de conclusiones críticas respecto al aspecto jurídico que demanda el tema.

\section{LOS COMPROMISOS NO ATENDIDOS}

Dentro de los objetivos principales que debe cumplir el Estado están la seguridad social, la alimentación, la vivienda, la salud y el empleo, dichos objetivos se encuentran inscritos en la primera parte de la Carta Magna, tradicionalmente llamada dogmática, donde sus preceptos incluso 
se conocieron en un principio como "garantías individuales", actualmente titulándose ese apartado como "De los Derechos Humanos".

El origen de la Constitución federal tuvo como finalidad proteger estos principios, pues pretendía atender las demandas sociales que provocaron el movimiento armado conocido como Revolución Mexicana. A pesar de las críticas que los estudiosos han hecho a dicho movimiento, no se puede negar que la Constitución sí plasmó y aseguró los derechos de las personas.

De esta forma se sentaron las bases para que se transitara hacia el llamado Estado de bienestar social, en el cual se asume la responsabilidad de brindar un gran número de servicios, pues existe el convencimiento de procurar la prosperidad de sus habitantes con una intervención directa ${ }^{1}$. Una de las estrategias tradicionales es el integrarlos al sector productivo, puesto que con el empleo se pueden cubrir las otras necesidades ${ }^{2}$.

Si bien el Estado crea fuentes directas de trabajo en el sector burocrático ${ }^{3}$, actualmente no es el mayor generador, pues más bien se encarga de perfeccionar las condiciones necesarias para que esto sea posible con la participación del capital privado y sus inversiones. Existen alrededor de 30 millones de empleos formales, de

1. Conforme al artículo 4 de la Constitución Política de los Estados Unidos Mexicanos: “... Toda persona tiene derecho a la protección de la salud. La ley definirá las bases y modalidades para el acceso a los servicios de salud y establecerá la concurrencia de la Federación y las entidades federativas en materia de salubridad general, conforme a lo que dispone la fracción XVI del artículo 73 de esta Constitución... Toda familia tiene derecho a disfrutar de vivienda digna y decorosa. La ley establecerá los instrumentos y apoyos necesarios a fin de alcanzar tal objetivo... Los niños y las niñas tienen derecho a la satisfacción de sus necesidades de alimentación, salud, educación...Los ascendientes, tutores y custodios tienen la obligación de preservar y exigir el cumplimiento de estos derechos y principios. El Estado otorgará facilidades a los particulares para que coadyuven al cumplimiento de los derechos de la niñez..."

2. Conforme al artículo 5 de la citada Constitución: "A ninguna persona podrá impedirse que se dedique a la profesión, industria, comercio o trabajo que la acomode, siendo lícitos. El ejercicio de esta libertad solo podrá vedarse por determinación judicial, cuando se ataquen los derechos de tercero, o por resolución gubernativa, dictada en los términos que marque la ley, cuando se ofendan los derechos de la sociedad. Nadie puede ser privado del producto de su trabajo, sino por resolución judicial... Tampoco puede admitirse convenio en que la persona pacte su proscripción $o$ destierro, o en que renuncie temporal o permanentemente a ejercer determinada profesión, industria o comercio..."

3. De hecho, el aparato burocrático se vio fuertemente reducido en México hace algunos años, durante el periodo neoliberal. los cuales solo un poco más de dos millones son plazas gubernamentales (Flores, 2016).

El modelo que propusieron los políticos denominados "tecnócratas" debía reducir al máximo la presencia del gobierno para que solo realizara algunas actividades elementales. "La primera característica generalmente atribuida al tecnócrata político, como aquí lo llamaremos, es que adquirió cierto conocimiento especializado que lo capacita para la posición en la cual tiene responsabilidades" (Ai Camp. 2010, p. 84). Como bien dice Roderic Ai Camp: "La capacidad, si es que ha de tener algún valor para comprender la función y definición del tecnócrata político, y para que pueda ser comprobada empíricamente, debe ser examinada en un largo periodo de tiempo para que se hagan visibles las tendencias en este tipo de liderazgo" (p. 84).

En dicho proyecto económico, el compromiso de generar competitividad y de asumir los riesgos ahora era responsabilidad de la iniciativa privada. Por lo tanto, el Estado debía construir, se decía, el derrotero para la globalización y así el país fuera atractivo a los ojos de las transnacionales y poder transformar a la sociedad y llevarla a la industrialización para crecer con equidad, seguridad y prosperidad.

La realidad resultó ser otra, pues la iniciativa privada no erradicó la pobreza, sino todo lo contrario, los índices se elevaron aún más. El proyecto político no fue capaz de dar respuesta a la problemática, de hecho, se ha agravado, pues las desventajas para la clase trabajadora son la poca certeza laboral y los bajos salarios.

La libertad que tuvo la iniciativa de libre mercado quedó plasmada en el término laissez faire $^{4}$, una libertad que solo se inscribió en el terreno económico y que benefició con creces a la clase empresarial portentosa, no así a las pequeñas y medianas empresas y a los trabajadores, quienes vieron un retroceso en todas sus conquistas laborales.

Dentro de los retrocesos, en materia económica, podemos mencionar que las grandes empresas encontraron la manera de eludir

4. Expresión francesa, que completa dice: "Laissez, faire, laissez passer" (dejar hacer, dejar pasar; es decir: permitid, tolerad toda iniciativa). Enciclopedia Jurídica. (2020). 
impuestos, lo cual afectó directamente a la implementación de programas sociales, si bien es cierto que no terminaban con la pobreza, al menos la sobrellevaban.

Cabe mencionar que no deja de ser consecuencia de lo anterior que "México ocupe el último lugar de la OCDE en recaudación de impuestos. Mientras la recaudación tributaria promedió 13.6\% del PIB en México en el período 2013-2017, en los países miembros de la OCDE dicho porcentaje fue de 33.4\%. Ante ello, es importante combatir la evasión y elusión fiscal a través de la reducción de tratamientos fiscales especiales, la adopción de buenas prácticas internacionales, el fortalecimiento de la cultura del cumplimiento de las obligaciones fiscales y de la promoción del uso de las tecnológicas de la información que faciliten el cumplimiento de obligaciones ante el fisco" (Clavellina, et al, 2019).

"El país enfrentaba nuevas circunstancias internas y externas, la mayoría de las reformas de nuestra Revolución han agotado sus efectos y no son ya la garantía del nuevo desarrollo que exige el país. Debemos, por ello, introducir cambios en el Estado, promover Conferencias magistrales nuevas formas de organizar la producción y crear nuevos esquemas de participación y de relación política" (Aguilar, 1994, p. 189).

Si bien el tema se llegó a considerar, nunca se debe de perder de vista que la figura del Estado fue creada para organizar políticamente a la sociedad y que el gobierno debe cumplir con la función dirigir, controlar y administrar con una conducción hacia el interés y bienestar general.

\section{LOS INSTRUMENTOS INTERNACIONALES DE DERECHOS ECONÓMICOS, SOCIALES Y CULTURALES Y LA REGULACIÓN EN MÉXICO DE LAS MICRO, PEQUEÑAS Y MEDIANAS EMPRESAS}

Los derechos humamos se dividen en diferentes categorías, a saber: civiles, políticos, económicos, sociales y culturales. Podemos encontrar un catálogo de los derechos humanos en la Declaración Universal de los Derechos Humanos, pero también se incluyen otros instrumentos legales $y$, refiriéndonos en específico a los derechos económicos, sociales y culturales, estos están protegidos por diversos tratados internacionales y regionales, así como en las constituciones nacionales. Existe el Pacto
Internacional de los Derechos Económicos, Sociales y Culturales (PIDESC), aprobado en 1996, es el tratado más exhaustivo que estipula a nivel internacional la protección de tales derechos, contiene un catálogo de derechos y establece que estos deben ser reconocidos por los Estados.

El pacto expresa el derecho a tener condiciones equitativas y satisfactorias de trabajo. En ese sentido, dentro del campo de empresas y derechos humanos, la obligación de respeto implica que los Estados deban abstenerse de desplegar conductas vinculadas a actividades empresariales que contravengan el ejercicio de los derechos humanos.

México, es un Estado parte en este Pacto, y ha de observar y velar por su cumplimiento, al igual que debe promocionar estos derechos y tener en cuenta las circunstancias nacionales. Se supone que a través de la ratificación de los pactos y tratados internacionales, los gobiernos se comprometen a tomar medidas y tener leyes internas que sean compatibles con las obligaciones y deberes que dimanan de los mismos.

En México ha sido difícil ubicar correctamente a través del tiempo a las micro, pequeñas y medianas empresas, puesto que las variables e indicadores que se han tomado en cuenta para clasificarlas han cambiado constantemente; de manera tradicional, se han utilizado parámetros económicos y contables para clasificarlas, tales como: el número de trabajadores, el total de ventas anuales, los ingresos y los activos fijos de las empresas.

Sin embargo, podemos mencionar que el sector empresarial, particularmente el relacionado con la micro, pequeña y mediana empresa (MYPYMES) se encuentra regulado por:

- La Constitución Política de los Estados Unidos Mexicanos.

- La Ley de Cámaras Empresariales y sus Confederaciones.

- La Ley de Comercio Exterior.

- La Ley de Concursos Mercantiles.

- La Ley de la Inversión Extranjera.

- La Ley para el desarrollo de la competitividad de la micro, pequeña y mediana empresa.

- Las Leyes Fiscales. 
La Constitución Política de los Estados Unidos Mexicanos establece en su artículo 25 la obligación del Estado para planear, conducir, coordinar y orientar la economía nacional y llevar a cabo la regulación y fomento de las actividades que demande el interés general, como es el caso de las MIPYMES.

La Ley Orgánica de la Administración Pública Federal (Art. 34, 2020), define las funciones y atribuciones de los organismos de la Administración Pública Federal, como es el caso de la Secretaría de Economía quien regula y fomenta el desarrollo de las empresas.

La Ley de Cámaras Empresariales y sus Confederaciones (Art. 1ํ) norma la constitución y funcionamiento de las Cámaras de Comercio, de Industria y de las Confederaciones que las integran, así como al Sistema de Información Empresarial Mexicano (Diario Oficial de la Nación, 2019).

Se dispone en México de la Ley para el desarrollo de la competitividad de la micro,

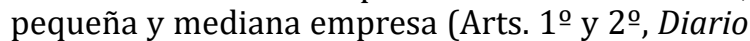
Oficial de la Nación, 2019), que en el artículo primero establece que esta ley tiene por objeto promover el desarrollo de la economía nacional, a través del fomento de creación de las empresas ya antes mencionadas; asimismo, el apoyo para su viabilidad, competitividad, sustentabilidad $\mathrm{y}$ productividad. Al igual que, aumentar su participación en los mercados.

Aunado a lo anterior menciona que la finalidad principal es la de fomentar el empleo, el bienestar económico y social de las micro, pequeñas y medianas empresas $y$, que el encargado de desarrollar los programas de apoyo para las empresas es la Secretaría de Economía.

Esto corrobora que el Estado Mexicano conforme a las disposiciones internacionales $\mathrm{y}$ nacionales tiene como obligación proporcionar el apoyo suficiente a dichas empresas, ya que el desarrollo de la economía depende bastante de las mismas. De igual forma deben ser apoyadas en afan de procurar el bienestar y mejora de las condiciones de los trabajadores que en estas laboran.

Conforme a lo expuesto, se puede afirmar que los instrumentos internacionales de derechos económicos, sociales y culturales son el parametro de acción en materia económica y social para los Estados ratificantes.

Los derechos económicos, sociales y culturales (DESC) tiene como finalidad cubrir cuestiones básicas de todo ciudadano como, por ejemplo: el trabajo, la seguridad social, la educación, la alimentación, la salud, el agua, la vivienda, la cultura, etc.

Actualmente el crecimiento económico representa solución a diversos problemas en México. Las pequeñas y medianas empresas de la industria del calzado de la zona de El Bajío, representan un área de oportunidad para el crecimiento económico, por tanto, resulta necesario resaltar aquello que disponen el ordenamiento jurídico nacional y los instrumentos internacionales en materia de derechos económicos, sociales y culturales, pues da lugar a que, además de que se dé un crecimiento económico, se observa la protección de los derechos humanos en el país.

Para lograr el tan anhelado crecimiento económico de la industria en México se debería de favorecer al sector privado para el fortalecimiento del comercio exterior (exportación); sin embargo, esto no se ha visto reflejado y, por el contrario, los modelos económicos han implementado políticas alejadas a observar y cumplir con lo dispuesto por los Derechos Económicos, Sociales y Culturales (DESC), lo que ha reportado un descenso económico.

\section{LA NECESIDAD DE IMPLEMENTAR ESTRATEGIAS EFECTIVAS}

La Organización para la Cooperación y el Desarrollo Económico (OCDE) afirma que la informalidad se concentra en empresas muy pequeñas, las cuales generan una desigualdad a nivel individual, pero esto no solo se refleja a nivel regional, donde es palpable esa diferencia de economías entre el sur del país, donde predomina una baja productividad, con el norte y el centro, que se caracterizan por tener una mayor productividad en los distintos sectores económicos.

Las políticas económicas que se han puesto en marcha en México no han sido las adecuadas, pues no han logrado alcanzar los resultados esperados, es decir, las condiciones apropiadas 
para crear fuentes de empleo permanentes y bien remuneradas. Hoy en día los jóvenes no tienen oportunidades de trabajo y quien lo consigue es para sumarse al sistema del empleo informal, ya que si estos laboran una de las micro, pequeñas y medianas empresas de la industria en México que se encuentran en la informalidad, ello repercute en que los trabajores no creen ninguna relación laboral ni de derecho frente a las mismas.

De hecho, el escenario se complicó al grado de tener grandes desventajas para los trabajadores, como pueden ser la poca certeza laboral y la proliferación del subempleo, además del ya mencionado trabajo informal, que ofrece una aparente zona de seguridad, en la cual se refugian muchas personas. A esto se le suma que algunas de estas actividades se encuentran inscritas en la ilegalidad.

"El subempleo aglomera la baja calidad del trabajo desde la oferta de mano de obra es decir, en el subempleo se encuentran los trabajadores que no se encuentran satisfechos con el trabajo que poseen. Partiendo de esto, se puede establecer que este tiene una relación intrínseca con la informalidad, ya que a partir de la relación de estas dos se habla de calidad del empleo" (Rodríguez y Calderón, 2015, pp. 41-58).

La imposibilidad de conseguir que México sea económicamente competitivo a nivel mundial vuelve inviable los proyectos de modernización y competitividad a escala global del país, pues la informalidad contribuye al poco crecimiento económico, lo que se traduce en una sociedad poco igualitaria y excluyente y la atención de dichas desigualdades se reducen por el hecho de que la base tributaria es reducida y quienes pudieran ensancharla se mueven en este espectro que bien podríamos llamar economía marginal.

El trabajo formal ayuda claramente a mejorar las condiciones de vida de las personas, pero además también es vital para el mejoramiento de las condiciones del Estado mismo, pues gracias a este es posible mejorar la captación de recursos a través de las inversiones o por vía de impuestos, lo cual no ocurre como debiera ser, puesto que México es el país de la OCDE que menos recursos capta a través de este rubro. Los ingresos fiscales representaron $16.2 \%$ del Producto Interno Bruto al cierre del 2017, una reducción respecto a 16.6\% del año previo (Morales, 2018).
México es un país desigual, en el cual se torna necesario elevar el nivel educativo y atacar la deserción escolar; consolidar el sistema de seguridad social; buscar la línea de bienestar con un salario mínimo digno; atacar la corrupción a través de mecanismos efectivos de transparencia y rendición de cuentas; tener en cuenta a los estados del sur, los más pobres del país y, atender a través de políticas públicas la necesidad de apoyar a la industria nacional, con la finalidad de que la clase trabajadora se inserte en el mercado internacional con empleos dignos y de calidad (Alcocer, 2020).

La industria es robusta y se cuenta con una gran capacidad de producción, al grado de haber sabido aprovechar las vicisitudes entre China y Estados Unidos, para convertirse en el primer trimestre del 2020 como el principal socio comercial de la Unión Americana.

El INEGI (2020) reporta en un comunicado de prensa, , información sobre la balanza comercial con diferentes mercados externos, en estos términos: "La información oportuna de comercio exterior de febrero de 2020 indica un superávit comercial de 2,911 millones de dólares, saldo que se compara con el superávit de 1,340 millones de dólares obtenido en igual mes de 2019. En los primeros dos meses de este año, la balanza comercial presentó un superávit de 495 millones de dólares".

Por otra parte, la prensa especializada en finanzas y negocios destacó el comercio entre México y Estados Unidos durante el primer trimestre del 2020, con una participación conjunta en la que las exportaciones de los Estados Unidos alcanzaron un total de 72 mil 807 millones de dólares, mientras que sus importaciones ascendieron a 103 mil 325 millones de dólares, según datos de la Oficina del Censo de Estados Unidos (2020).

El periódico El País señala que "México se ha convertido por primera vez en el primer socio comercial de Estados Unidos, de acuerdo con cifras que ha presentado esta semana el Gobierno estadounidense". Es obvio que la guerra comercial con China y la pandemia provocada por el Covid-19 plantearon un nuevo entorno, el cual hasta el momento ha sido aprovechado por México. 
Tabla 1. Comercio estadounidense con México

\begin{tabular}{|c|c|c|}
\hline MES & EXPORTACIONES EUA & IMPORTACIONES EUA \\
\hline Enero & $20,834.00$ & $28,331.80$ \\
\hline Febrero & $19,397.50$ & $29,058.10$ \\
\hline Marzo & $20,048.1$ & $30,106.60$ \\
\hline Abril & $12,527.50$ & $15,829.10$ \\
\hline Total & $72,807.00$ & $103,325.60$ \\
\hline
\end{tabular}

Fuente: Elaboración propia a partir de la información que se obtiene del Bureau del Censo de los Estados Unidos, Comercio exterior, Comercio de bienes con México. Todas las cifras están expresadas en millones de dólares. Las sumas pueden variar debido a los redondeos.

La balanza comercial nos indica que las exportaciones son mayores que las importaciones, lo cual es una noticia histórica para el país. Sin embargo, se debe saber leer lo que ocurre en los distintos escenarios, pues según el reporte de la Oficina del Censo de Estados Unidos, este deterioro en la balanza comercial fue consecuencia de la pandemia provocada por el Covid-19. Esta información fue difundida a través de un tuit por la embajada de México en Estados Unidos.

Se observa que es posible obtener crecimiento sin tener que recurrir al proteccionismo, pues hoy en día este tipo de posturas en lugar de beneficiar solamente afectaría al intercambio comercial. De hecho, desde hace mucho tiempo, México se ha alejado de esas prácticas comerciales, en las cuales cerraba sus fronteras y aplicaba de manera desmedida aranceles a todos los productos provenientes del exterior.

Además, hoy en día, existen una cantidad importante de tratados y acuerdos comerciales con distintos países, lo que significa que se han signado compromisos internacionales, que deben ser respetados, pues debe prevalecer el principio pacta sunt servanda 5 . De hecho, otra opción podría ser el diversificar esa baraja de tratados de libre comercio, de los cuales nuestra nación forma parte, sin descuidar el T-Mec ${ }^{6}$ y así aprovechar al máximo la apertura comercial.

5. (Derecho General), (Derecho Internacional Público) "Los pactos hay que guardarlos". Locución latina que proclama el principio según el cual los tratados y, más en general, los contratos deben ser respetados por las partes que los han concluido.

6. Tratado Comercial entre México Estados Unidos y Canadá, con la entrada en vigor del instrumento a partir del $1^{\circ}$ de julio de
México cuenta con una red de 12 tratados de libre comercio con 46 países, 32 acuerdos para la promoción y protección recíproca de las Inversiones con 33 países y, 9 acuerdos de alcance limitado. Además, México participa activamente en organismos y foros multilaterales y regionales como la Organización Mundial del Comercio (OMC), el Mecanismo de Cooperación Económica Asia-Pacífico (APEC), la Organización para la Cooperación y Desarrollo Económicos (OCDE) y la Asociación Latinoamericana de Integración (ALADI) (Secretaría de Economía, Acciones y Programas, 2015).

Históricamente, se está ante una situación inédita, en el sentido que ningún país puede estar al margen de los demás sin sufrir consecuencias económicas y en este punto queremos retomar al sociólogo Marshall Mac Luhan (1990), y aplicar sus afirmaciones en un sentido mucho más amplio, pues somos habitantes de una aldea global, donde la interconectividad que experimentamos es a escala global, pues todos los cambios sociales ocurridos en cualquier punto del planeta tendrán repercusiones en nuestro entorno (p. 93) ${ }^{7}$.

Tal parece que el mundo es más pequeño, el desarrollo tecnológico ha sido responsable de la eficiencia en el transporte, pero también de las comunicaciones, pues el internet hace posible que la comunicación sea inmediata. Aunado a lo anterior, también las transacciones económicas y transferencias se pueden llevar a cabo de manera

$\overline{2020 \text { se asegura la competitividad de la región e inicia una nueva }}$ etapa que fortalecerá el comercio y la inversión en América del Norte.

7. MACLUHAN, Marshaly Powers, B.R. La aldea global, Barcelona, Gedisa, 1990, p. 93. 
electrónica, lo que le da un sentido de inmediatez al comercio mundial.

\section{GLOBALISMO, GLOBALIDAD Y GLOBALIZACIÓN}

Pudiera parecer un contrasentido lo que hasta aquí se ha dicho, pero cabría afinar ciertos puntos para que esas aparentes contradicciones queden aclaradas, para lo cual nos apoyaremos en lo que afirma el sociólogo Ulrich Beck, quien dice que existe una falacia de aquellos que pretenden utilizar en el mismo sentido, como si se trataran de sinónimos, dos conceptos que tienen sus diferencias.

Beck aclara que globalismo, globalidad y globalización no son lo mismo. En el primero de ellos, se hace referencia a una ideología en la cual el mercado mundial sustituye al quehacer político, todo se reduce al aspecto económico. Por globalidad se debe entender a la situación que impera hoy en día, es decir, la situación de que ningún país puede vivir al margen de los demás y, finalmente, denomina globalización al proceso que experimentan los Estados nacionales al entremezclarse (2004, pp. 25-26).

Es palpable la falta de desarrollo industrial en ciertas regiones geográficas, lo que contrasta con otras partes de la nación, donde el desarrollo económico está consolidado, pues ya existen industrias afianzadas, lo anteriormente descrito demuestra que México no es un país homogéneo en su desarrollo económico, pues existe una gran diversidad de actividades económicas.

Una región del país que ha experimentado un crecimiento importante es El Bajío mexicano, dicha área geográfica comprende los Estados de Querétaro, Guanajuato, Aguascalientes y la zona oriente de Jalisco, mejor conocida como Los Altos de Jalisco. Hay quienes incluyen a los Estados de Michoacán y San Luis Potosí. Y se ubica en la altiplanicie mexicana (Mejía y Nava, 2017, p. 40).

La apertura de mercados en la década de los noventa afectó a diversos sectores productivos de la nación y la rama regional del calzado no fue la excepción, siendo los más afectados los medianos y pequeños productores, pues al no contar con la asesoría adecuada y la participación del gobierno apoyándolos y direccionando su consolidación en un ámbito internacional, no pudieron implementar las herramientas financieras adecuadas para hacer frente a la apertura de fronteras comerciales.

De hecho, tuvieron que ir adecuando y sorteando los problemas como se les vinieran presentando, pues en un mundo globalizado los competidores ya no solamente, son aquellas empresas que son físicamente visibles. Ahora se está en un mundo virtual, la aldea global, donde se debe competir con cualquier otra empresa similar ubicada en cualquier lugar del planeta.

Lo anteriormente señalado es motivo suficiente para transformar las cualidades en ventajas competitivas, lo cual hace importante el diseño de medidas económicas que permitan a las pequeñas y medianas empresas dedicadas a la fabricación y distribución de calzado acceder sin restricciones a los beneficios que se han implementado para los grandes industriales de este sector.

\section{ESTRATEGIAS COMERCIALES PARA LA} INDUSTRIA DEL CALZADO EN MÉXICO

Se vuelve de vital relevancia que los gobiernos, tanto el federal como de los distintos Estados de El Bajío, no solamente permitan, sino que también diseñen programas inspirados en este sector específico $y$, así evitar que se queden al margen por no cumplir con los perfiles que sí tienen los grandes productores que por lo regular son externos.

Como se señaló en el primer apartado del presente escrito, las decisiones políticas que se advierten en México no han demostrado compatibilidad con los DESC, frente a esto es importante cuestionarnos si estas decisiones han implicado una herramienta o una barrera para las pequeñas y medianas empresas mexicanas de la industria del calzado de la zona de El Bajío que apenas se estaban consolidando. El comportamiento de las empresas ha cambiado paulatinamente, un número mayor de éstas realizan actividades de carácter internacional, pero esto no ha repercutido en que las empresas mexicanas tengan una sólida presencia en la escena internacional (Fillis, 2001, pp. 767-783).

La propuesta de Adam Smith (1794) para el correcto funcionamiento de una economía de mercado, es que el Estado debe regular e intervenir para garantizar el acceso a todos los agentes económicos a los mecanismos 
de mercado, posibilitando una economía de mercado abierta a todos los hombres (pp. 203207). Teniendo en cuenta que la desigualdad en el acceso al financiamiento y en las condiciones de acceso para las empresas de menor tamaño constituye un eje de la reproducción de la heterogeneidad estructural y desigualdades sociales (Ferraro, 2011, p. 53). Por lo anterior, se duda de la compatibilidad entre los últimos modelos económicos implementados en México y lo previsto en los instrumentos internacionales de derechos económicos, sociales y culturales.

Aplicar los instrumentos internacionales en materia de derechos económicos, sociales y culturales que protegen derechos humanos, es uno de los deberes que el gobierno debe cumplir para su protección y promoción. Las estrategias implementadas deben favorecer al sector privado para el fortalecimiento del comercio exterior (exportación).

La ya mencionada Ley para el desarrollo de la competitividad de la micro, pequeña y mediana empresa, en el artículo tercero nos muestra una tabla en la cual se señala la estratificación por número de trabajadores, y de igual manera desglosa como se manejan los apoyos y a quienes van destinados y de qué manera. Sin embargo, esto no basta si no se tienen resultados medibles y cuantificables en los beneficiados, como: el incremento en ventas, el incremento en empleo, la mejora de la productividad, el aumento de la competitividad, un mejor posicionamiento en el mercado, el acceso a nuevos mercados, el incremento de la calidad de los productos y/o servicios, una mayor cobertura, mayor facilidad para hacer negocios o cualquier otro que contribuya al fortalecimiento de la economía nacional.

Generar mediante la colocación eficaz y la selección, los mejores proyectos presentados al Fondo PYME $^{8}$ a través de un proceso de evaluación sólido y transparente, una gradual y firme transformación de los emprendedores y de

8. El Fondo de Apoyo para la Micro, Pequeña y Mediana Empresa (FONDO PYME) es un instrumento instaurado por el gobierno mexicano a través de la Secretaría de Economía, que busca apoyar a micro, pequeñas y medianas empresas y a los emprendedores, con el propósito de promover el desarrollo económico nacional, otorga apoyos de carácter temporal a programas y proyectos que fomenten la creación, desarrollo, viabilidad, productividad, competitividad y sustentabilidad de las empresas en particular las de menor tamaño. las micro, pequeñas y medianas empresas de la industria del calzado en el país, para que las micro empresas se transformen en pequeñas empresas, las pequeñas en medianas y las medianas en grandes, favoreciendo así el crecimiento sostenido de la economía mexicana.

Crear mecanismos eficientes de generación y distribución de los apoyos que garanticen el ejercicio de los recursos y la llegada de estos a los beneficiarios.

Los objetivos del Fondo PYME deberán desarrollarse de manera eficaz y oportuna a fin de consolidar el surgimiento, fortalecimiento y competitividad de los emprendedores y de las micro, pequeñas y medianas empresas del país, la transformación competitiva de sectores y regiones en el país, así como la inversión productiva generadora de empleos y bienestar.

Con estas instancias, para generar economía está implícito que a cada uno de los emprendedores se les facilite, en cuanto ámbito de contribución, que sea congruente con el principio de proporcionalidad; también, que de ser necesario se amplié un fondo de modelo aseguradora para prevenir escases en casos de caída de moneda o imprevistos económicos, los cuales pueden absorber los gastos en los primeros meses y que así mismo se vuelva a flote.

Apoyando a las empresas del país que aunque parezcan pequeñas, si se les fortalece pueden ser generaradoras de empleos, pero para esto se requiere que sean competitivas. Las micro, pequeñas y medianas empresas desempeñan un papel importante en el desarrollo económico de las naciones, tanto en los países industrializados, como en los de menor grado de desarrollo; como es el caso de México.

En cuanto a la industria del calzado en México, es un error quererle competir de frente a China con productos de bajo costo, esa fortaleza les pertenece a ellos, la manera viable de contrarrestar esta debilidad es con productos de calidad, los cuales se puedan exportar no solo al mercado norteamericano, sino al asiático. Al mercado chino se le pueden ofrecer productos de calidad.

Una cantidad importante de este producto corresponde al denominado calzado de línea 
blanca, es decir el más económico. En cambio, también existen otras líneas, las de gama media alta y alta, aquí el producto que se comercializa es el denominado de marca y ostenta una mayor calidad y por lo tanto los precios no son tan bajos.

Entonces, se tiene una estrategia comercial importante, abandonar de manera paulatina la fabricación del calzado de línea blanca, puesto que hay una gran inviabilidad de competir con este tipo de mercancía de importación y, enfocarse en la competencia de manera significativa del calzado clasificado como de gama media alta y alta.

Por lo tanto, es primordial aumentar los recursos que se asignan a potenciar las marcas de los productos que comercializan los pequeños y medianos productores, al mismo tiempo se debe acrecentar la calidad del producto final. En segundo lugar, también debe mejorar la calidad de los canales de distribución, no solo a nivel nacional, sino también internacional.

Dentro de estas estrategias, en las cuales grosso modo se habla de las medidas urgentes e imperantes que deben tomarse para generar estrategias que impulsen al sector zapatero. Sin embargo, no se debe descuidar el mercado interno, pues este ayuda a satisfacer necesidades e incluso la derrama económica puede ser más importante que la generada por los productos de importación.

De hecho, una buena alternativa sería copiar el esquema japonés, en el que el gobierno creó políticas industriales proteccionistas, el aislamiento del mercado extranjero y la reestructuración financiera. Se fomentó la cooperación entre el pueblo y gobierno, cooperación entre empresarios y trabajadores y una distribución equitativa de los beneficios derivados del aumento de la productividad (Rodríguez, 2020).

No resulta desfasado, por como está la situación de la industria del calzado, que el gobierno mexicano intervenga como rector en la economía y quede intacta la tasa arancelaria para el rubro de la importación del calzado, puesto que la reducción de los aranceles perjudicaría directamente a la industria del calzado. No olvidemos que el pasado 31 de enero de 2019 se publicó en el Diario Oficial de la Federación (DOF) la disminución arancelaria para la importación de calzado al país.

Esto aparentemente beneficia en primera instancia a los consumidores, pero a mediano y largo plazo afecta a los fabricantes y a la industria del calzado nacional en general, pues será desplazada por el calzado de importación, lo que en un periodo no muy largo provocará la pérdida de puestos de trabajo en el ramo. Las cámaras que representan a la industria textil y del calzado mexicanas prevén una pérdida fiscal aproximada conjunta de unos 4 mil 800 millones de pesos anuales por la menor recaudación (Reuters, 2019). Por lo cual es urgente implementar estrategias comerciales para la industria del calzado en México.

\section{CONCLUSIONES}

No existe ningún Estado en el mundo donde el gobierno haya renunciado a intervenir en las tareas económicas en su totalidad. Este "dejar hacer, dejar pasar" aplicado de manera total es conocido como capitalismo en estado puro, es decir, el Estado, de manera legal, es totalmente ajeno de la economía y del mercado. La realidad ha demostrado que esto no es posible, pues durante las grandes crisis del sistema capitalista siempre es el Estado quien llega al rescate de las economías resquebrajadas.

Se requiere el compromiso de las empresas, pero también de las tres esferas de gobierno, federal, local y municipal, de cada uno de los Estados dedicados a la rama de la industria zapatera, puesto que la presente problemática no solo se trata del comercio que realizan ciertos empresarios, sino que el impacto es a nivel regional, ya que influye directamente sobre el desarrollo de la sociedad en su conjunto.

La región de El Bajío mexicano se distingue por ser el lugar donde floreció la industria del calzado en México, pero no solo eso, sino que llegó a ser de las zonas más productivas en todo el país.

Sin embargo, las políticas de Estado que dejaron de proteger a las pequeñas y medianas empresas de la industria del calzado en México, han provocado una gran brecha en el camino a la competitividad comercial.

Por todo lo anterior, México debe desarrollar $\mathrm{y}$ posteriormente implementar una serie de 
medidas macroeconómicas como las que aquí se han expuesto y así permitir que no solo las grandes empresas privadas, sino también los pequeños y medianos productores desarrollen ventajas competitivas sin restricciones.

El Estado mexicano debe velar por observar y cumplir con lo dispuesto en los derechos económicos, sociales y culturales; y tener la capacidad de ser un actor importante en el ámbito internacional. Esto solo se logrará si se le proporciona a la industria nacional los mecanismos adecuados para exportar.

Fomentar el crecimiento económico de México al mitigar las diferentes formas de exclusión económica, logrando la inserción a las cadenas de valor de los sectores estratégicos de mayor dinamismo, implica realizar las adecuaciones necesarias, implementar estrategias comerciales acordes a la protección de los derechos humanos que prevén los instrumentos internacionales de derechos económicos, sociales y culturales (tratados, convenciones, pactos y protocolos) de los que México forma parte, situación de relevante importancia para constituir un núcleo más dinámico de inserción internacional, papel que debe tomar el Estado mexicano frente a la globalización.

Será sumamente frutifero que en el diseño e implemetación de estrategias comerciales para la industria del calzado en México, se tenga conciencia de que el apoyo a las micro, pequeñas y medianas empresas es primordial para su consolidación y permanencia, así como para el bienestar y estabilidad laboral de los trabajadores que en estas se desempeñan, pues solo así podríamos hablar de la eficacia del gobierno, para dirigir el rumbo de la economía del país.

\section{BIBLIOGRAFÍA}

- Ai Camp, R. (2010). El tecnócrata en México. Revista Mexicana de Opinión Pública, (9): 83100, Disponible en: http://www.redalyc. org/articulo.oa?id=487456193007.

- Aguilar, L. F. (1994). El liberalismo social del presidente Salinas de Gortari: una interpretación. Revista Mexicana de Ciencias Políticas y Sociales 39 (156), p.p. 189 221. Disponible en: http://www.revistas. unam.mx/index.php/rmcpys/article/ view/49956/44922

- Alcocer, G. M. (2020). México Justo: políticas públicas contra la desigualdad. Disponible en https://n9.cl/95oqb

- Beck, U. (2004). ¿Qué es la globalización? Falacias del globalismo, respuestas a la globalización. Ciudad de México: Paidós.

- Clavellina M., J. L., et al. (2019). Alternativas para reducir la evasión y elusión de impuestos de las Empresas 'Factureras' o 'Fantasmas'”. En Serie Notas Estratégicas (60). Disponible en línea: http://bibliodigitalibd.senado.gob.mx/ handle/123456789/4575

- Constitución Política de los Estados Unidos Mexicanos. México. 2020.

- Enciclopedia jurídica. (2020). Disponible en http://www.enciclopedia-juridica.com/d/ laissez-faire/laissez-faire.htm

- Ferraro, C. (2011). Eliminando barreras: El financiamiento a las pymes en América Latina. Madrid: CEPAL.

- Flores, Z. (13 de septiembre de 2016). Bajan burocracia los 3 niveles de gobierno. El Financiero. Disponible en https:// www.elfinanciero.com.mx/economia/ bajan-burocracia-los-niveles-de-gobierno

- Fillis, I. (2001). Small firm Internationalization: an investigative survey and future research directions. Journal of management decision, 39(9):767-783.

- Instituto Nacional de Estadística y Geografía - INEGI, (27 de marzo de 2020). Comunicado de prensa 140/20, "Balanza comercial de mercancías de México". Disponible en 
https://www.inegi.org.mx/contenidos/ saladeprensa/boletines/2020/balcom_o/ balcom_02020_03.pdf

- Bureau del Censo de los Estados Unidos. (2020). Disponible en https://www.census. gov/foreign-trade/balance/c2010.html

- Ley de Cámaras Empresariales y sus Confederaciones. (12 de abril de 2019). Diario Oficial de la Federación.

- Ley Orgánica de la Administración Pública Federal. (22 de enero de 2020). Diario Oficial de la Federación.

- Ley para el desarrollo de la competitividad de la micro, pequeña y mediana empresa, (13 de agosto de 2019). Diario Oficial de la Federación.

- Macluhan, M., y Powers, B. R. (1990). La aldea global, Barcelona Gedisa.

- Mejía, E., y Nava, E. F.. (2017) "El Bajío mexicano, estudios recientes", Ciudad de México: Sociedad Mexicana de Antropología.

- Morales, Y. (05 de diciembre de 2018). México ocupa último sitio en recaudación en zona OCDE. El Financiero, Disponible en: https://n9.cl/051kb

- Pacto Internacional de Derechos Económicos, Sociales y Culturales (PIDESC). 16 de diciembre de 1966.
- Reuters. (01 de febrero de 2019). México no renueva salvaguarda a acero; reduce aranceles a textiles y calzado". Excélsior, Disponible en. https://n9.cl/xw9hc.

- Rodríguez A., E. (2007) El Gran Salto Económico de Japón de Posguerra. Revista académica Contribuciones a la Economía, (4). Disponible en http://www.eumed.net/ ce/2007a/era11.htm (10.03.2020).

- Rodríguez L., G. I., y Calderón D., M.A. (2015). La economía informal y el desempleo: el caso de la ciudad de Bucaramanga (Colombia). Innovar Revista de Ciencias Administrativas y Sociales 25 (55): 41-58. Disponible en. https://www.redalyc.org/ articulo.oa?id=81832838004

- Secretaría de Economía, Acciones y programas. (10 de mayo de 2015), Comercio Exterior/Países con Tratados y Acuerdos firmados con México. Disponible en https://www.gob.mx/se/acciones-yprogramas/comercio-exterior-paises-contratados-y-acuerdos-firmados-con-mexico

- Smith, A. (1794). Investigación de la naturaleza y causa de la riqueza de las naciones. Madrid: Oficina de la viuda e Hijos de Santander. 